\title{
Two New Families of Finsler Connections on Even-Dimensional Manifolds
}

\author{
H. R. Salimi Moghaddam \\ (Communicated by Murat TOSUN)
}

\begin{abstract}
Let $F^{2 n}=\left(M, M^{\prime}, F^{*}\right)$ be an even-dimensional pseudo-Finsler manifold. We construct an almost hypercomplex structure on any chart domain of a certain atlas of $M^{\prime}$ by using a considered nonlinear connection. Then by using the almost hypercomplex structure we define two new families of Finsler connections. Also we show that for any Finsler connection $\nabla$ there exists a linear connection $D$ such that the local almost hypercomplex structure is parallel with respect to it.
\end{abstract}

Keywords: pseudo-Finsler manifold; Finsler connection; almost hypercomplex structure.

AMS Subject Classification (2010): 53C60, 53B05, 53C15.

\section{Introduction}

A. Bejancu and H.R. Farran, in [1] and [2] , for a pseudo-Finsler manifold $F^{m}=\left(M, M^{\prime}, F^{*}\right)$ with a non-linear connection $H M^{\prime}$ and any two skew-symmetric Finsler tensor fields of type $(1,2)$ on $F^{m}$, introduced a notion of Finsler connections which named " $\left(H M^{\prime}, S, T\right)$-Cartan connections". After them in [3] we reconstruct the same Finsler connections by using almost complex structures.

On the other hand almost hypercomplex and hypercomplex structures which are important in differential geometry have many interesting and effective applications in theoretical physics. For example the background objects of HKT-geometry are hypercomplex manifolds. These spaces appear in $N=4$ supersymmetric model (see $[4,5]$.$) . Applications of Riemannian metrics on these spaces persuade us to study the geometry of Finsler$ metrics on them (see $[6,7]$ ).

In the present paper we study the relations between Finsler structures and almost hypercomplex structures in a different viewpoint. We use the almost hypercomplex structures to construct new Finsler connections on even-dimensional pseudo-Finsler manifolds . For this purpose we construct a local almost hypercomplex structure by using a considered non-linear connection. Then by using the almost hypercomplex structure we define two new families of Finsler connections. Also we show that for any Finsler connection $\nabla$ there exists a linear connection $D$ such that the local almost hypercomplex structure is parallel with respect to it.

\section{Preliminaries and notations}

Assume that $M$ is a real $m$-dimensional smooth manifold and $T M$ is the tangent bundle of $M$. Let $M^{\prime}$ be a nonempty open submanifold of $T M$ such that $\pi\left(M^{\prime}\right)=M$ and $\theta(M) \cap M^{\prime}=\emptyset$, where $\theta$ is the zero section of $T M$. Suppose that $F^{m}=\left(M, M^{\prime}, F^{*}\right)$ is a pseudo-Finsler manifold where $F^{*}: M^{\prime} \longrightarrow \mathbb{R}$ is a smooth function which satisfies the following conditions in any coordinate system $\left\{\left(\mathcal{U}^{\prime}, \Phi^{\prime}\right): x^{i}, y^{i}\right\}$ in $M^{\prime}$, :

- $F^{*}$ is positively homogeneous of degree two with respect to $\left(y^{1}, \ldots, y^{m}\right)$, i.e., we have

$$
F^{*}\left(x^{1}, \ldots, x^{m}, k y^{1}, \ldots, k y^{m}\right)=k^{2} F^{*}\left(x^{1}, \ldots, x^{m}, y^{1}, \ldots, y^{m}\right)
$$

for any point $(x, y) \in\left(\mathcal{U}^{\prime}, \Phi^{\prime}\right)$ and $k>0$. 
- At any point $(x, y) \in\left(\mathcal{U}^{\prime}, \Phi^{\prime}\right), g_{i j}$ are the components of a quadratic form on $\mathbb{R}^{m}$ with $q$ negative eigenvalues and $m-q$ positive eigenvalues, $0<q<m$ (see [1]).

Consider the tangent mapping $\pi_{*}: T M^{\prime} \longrightarrow T M$ of the projection map $\pi: M^{\prime} \longrightarrow M$ and define the vector bundle $V M^{\prime}=\operatorname{ker} \pi_{*}$. A complementary distribution $H M^{\prime}$ to $V M^{\prime}$ in $T M^{\prime}$ is called a non-linear connection or a horizontal distribution on $M^{\prime}$

$$
T M^{\prime}=H M^{\prime} \oplus V M^{\prime} .
$$

In local coordinates let $\left\{\frac{\delta}{\delta x^{i}}=\frac{\partial}{\partial x^{i}}-N_{i}^{j}(x, y) \frac{\partial}{\partial y^{j}}, \frac{\partial}{\partial y^{i}}\right\}, \quad(i, j=1 \cdots m)$ be the canonical basis for $T M^{\prime}$. Let $F^{m}=\left(M, M^{\prime}, F^{*}\right)$ be a pseudo-Finsler manifold. Then a Finsler connection on $F^{m}$ is a pair $F C=\left(H M^{\prime}, \nabla\right)$ where $H M^{\prime}$ is a non-linear connection on $M^{\prime}$ and $\nabla$ is a linear connection on the vertical vector bundle $V M^{\prime}$ (see [1]).

An almost hypercomplex manifold is a $4 n$-dimensional manifold $M$ with three globally-defined almost complex structures $J_{i}, i=1,2,3$, satisfying the quaternion identities

$$
\begin{aligned}
J_{1} J_{2} & =-J_{2} J_{1}=J_{3}, \\
J_{i}^{2} & =-I d_{T M}, \quad i=1,2,3 .
\end{aligned}
$$

Remark 2.1. From now on we suppose that $F^{2 n}=\left(M, M^{\prime}, F^{*}\right)$ is an even-dimensional pseudo-Finsler manifold. We use $h$ and $v$ for the projections on $H M^{\prime}$ and $V M^{\prime}$, respectively. Also we use $\delta_{i}$ and $\partial_{i}$ as $\frac{\delta}{\delta x_{i}}$ and $\frac{\partial}{\partial y_{i}}$ respectively.

Throughout the article, we use the following rules for the indices.

- The indicant $a$ is equal to 1 and 3, only.

- Latin indices (except the alphabets $a$ and $n$ ) run from 1 to $2 n$.

- Greek indices $\alpha, \beta, \gamma$ and $\theta$ run from 1 to $n$.

- Any repeated pair of indices (except the alphabets $a$ and $n$ ), provided that one is up and the other is down, is automatically summed.

- The matrix $\left(g^{i j}\right)$ is the matrix inverse of $\left(g_{i j}\right)$.

\section{New Finsler connections on even-dimensional manifolds}

In this section we construct two new families of Finsler connections on $M^{\prime}$. For this reason we consider an atlas on $M^{\prime}$, also we suppose that $H M^{\prime}$ is a non-linear connection on $M^{\prime}$. Then by using the non-linear connection we define an almost hypercomplex structure on any chart. After this step we define new Finsler connections on any chart by using the almost hapercomplex structure . Finally it is enough to paste the local connections by a partition of unity to have a connection on $M^{\prime}$.

Let $\mathcal{A}$ be an atlas on $M^{\prime}$ and $\left(\mathcal{U}^{\prime}, \Phi^{\prime}\right) \in \mathcal{A}$. We construct the following almost hypercomplex structure on $\mathcal{U}^{\prime}$ by using non-linear connection $H M^{\prime}$.

$$
\begin{array}{ccc}
J_{1}\left(\delta_{\alpha}\right)=\partial_{\alpha} & J_{2}\left(\delta_{\alpha}\right)=\delta_{n+\alpha} & J_{3}\left(\delta_{\alpha}\right)=-\partial_{n+\alpha} \\
J_{1}\left(\delta_{n+\alpha}\right)=-\partial_{n+\alpha} & J_{2}\left(\delta_{n+\alpha}\right)=-\delta_{\alpha} & J_{3}\left(\delta_{n+\alpha}\right)=-\partial_{\alpha} \\
J_{1}\left(\partial_{\alpha}\right)=-\delta_{\alpha} & J_{2}\left(\partial_{\alpha}\right)=\partial_{n+\alpha} & J_{3}\left(\partial_{\alpha}\right)=\delta_{n+\alpha} \\
J_{1}\left(\partial_{n+\alpha}\right)=\delta_{n+\alpha} & J_{2}\left(\partial_{n+\alpha}\right)=-\partial_{\alpha} & J_{3}\left(\partial_{n+\alpha}\right)=\delta_{\alpha}
\end{array}
$$

It is easy to show

$$
\begin{aligned}
J_{1}^{2} & =J_{2}^{2}=J_{3}^{2}=-I d_{T \mathcal{U}^{\prime}} \\
J_{3} & =J_{1} \circ J_{2}=-J_{2} \circ J_{1}
\end{aligned}
$$

Now by using $J_{1}$ and $J_{3}$ we construct two linear connections on $\mathcal{U}^{\prime}$ as follows.

Theorem 3.1. Let $\nabla$ be a Finsler connection on $\mathcal{U}^{\prime}$. The differential operators $D^{a}, a=1,3$, which are defined by

$$
D_{X}^{a} Y:=\nabla_{X} v Y-J_{a} \nabla_{X} J_{a} h Y \quad \forall X, Y \in \Gamma\left(T \mathcal{U}^{\prime}\right)
$$

are two linear connections on $\mathcal{U}^{\prime}$. Also $J_{1}$ and $J_{3}$ are parallel with respect to $D^{1}$ and $D^{3}$, respectively. 
Proof. The proof is easy so we omit it.

Let $T^{D^{a}}, a=1,3$, denotes the torsion tensor of $D^{a}$. It is simple to see,

$$
\begin{aligned}
T^{D^{a}}(X, Y)= & \left(\nabla_{X} v Y-\nabla_{Y} v X-v[X, Y]\right)- \\
& J_{a}\left(\nabla_{X} J_{a} h Y-\nabla_{Y} J_{a} h X-J_{a} h[X, Y]\right) \quad \forall X, Y \in \Gamma\left(T \mathcal{U}^{\prime}\right) .
\end{aligned}
$$

Now we can introduce two families of new Finsler connections by using $J_{1}$ and $J_{3}$.

Theorem 3.2. Suppose that $H M^{\prime}$ is a non-linear connection on $M^{\prime}$ and $S$ and $T$ are two arbitrary skew-symmetric Finsler tensor fields of type $(1,2)$ on $F^{2 n}$. Then there exists a unique linear connection $\nabla^{a}$ on $\mathrm{VU}^{\prime}$ satisfying the conditions:

1. $\nabla^{a}$ is a metric connection.

2. $T^{D^{a}}, S$ and $T$ satisfy,

$$
\begin{aligned}
& \text { i } T^{D^{a}}(v X, v Y)=S(v X, v Y), \\
& \text { ii } h T^{D^{a}}(h X, h Y)=J_{a} T\left(J_{a} h Y, J_{a} h X\right) .
\end{aligned}
$$

Proof. For any $X, Y, Z \in \Gamma\left(T \mathcal{U}^{\prime}\right)$ we define a linear connection $\nabla^{a}$ on $V \mathcal{U}^{\prime}$ by the following equations.

$$
\begin{aligned}
2 g\left(\nabla_{v X}^{a} v Y, v Z\right)= & v X(g(v Y, v Z))+v Y(g(v Z, v X))-v Z(g(v X, v Y)) \\
& +g(v Y,[v Z, v X])+g(v Z,[v X, v Y])-g(v X,[v Y, v Z]) \\
& +g(v Y, S(v Z, v X))+g(v Z, S(v X, v Y))-g(v X, S(v Y, v Z))
\end{aligned}
$$

and

$$
\begin{aligned}
2 g\left(\nabla_{h X}^{a} J_{a} h Y, J_{a} h Z\right)= & h X\left(g\left(J_{a} h Y, J_{a} h Z\right)\right)+h Y\left(g\left(J_{a} h Z, J_{a} h X\right)\right) \\
& -h Z\left(g\left(J_{a} h X, J_{a} h Y\right)\right)+g\left(J_{a} h Y, J_{a} h[h Z, h X]\right) \\
& +g\left(J_{a} h Z, J_{a} h[h X, h Y]\right)-g\left(J_{a} h X, J_{a} h[h Y, h Z]\right) \\
& +g\left(J_{a} h Y, T\left(J_{a} h Z, J_{a} h X\right)\right)+g\left(J_{a} h Z, T\left(J_{a} h X, J_{a} h Y\right)\right) \\
& -g\left(J_{a} h X, T\left(J_{a} h Y, J_{a} h Z\right)\right) .
\end{aligned}
$$

Now we show that $g$ is parallel with respect to $\nabla^{a}$. It is easy to see $J_{a} \circ v=h \circ J_{a}$ and $v \circ J_{a}=J_{a} \circ h$. After performing some computations for any $X, Y, Z \in \Gamma\left(T \mathcal{U}^{\prime}\right)$ we have,

$$
\begin{aligned}
\left(\nabla_{X}^{a} g\right)(v Y, v Z)= & \left(\nabla_{v X+h X}^{a} g\right)(v Y, v Z) \\
= & v X(g(v Y, v Z))-g\left(\nabla_{v X}^{a} v Y, v Z\right)-g\left(v Y, \nabla_{v X}^{a} v Z\right) \\
& +h X(g(v Y, v Z))-g\left(\nabla_{h X}^{a} v Y, v Z\right)-g\left(v Y, \nabla_{h X}^{a} v Z\right)=0 .
\end{aligned}
$$

So $\nabla^{a}$ is a metric connection.

Locally we set

$$
\begin{array}{rlrl}
\nabla_{\delta_{j}}^{1} \partial_{i} & =F_{i j}^{k} \partial_{k} & & \nabla_{\delta_{j}}^{3} \partial_{i}=\tilde{F}_{i j}^{k} \partial_{k} \\
\nabla_{\partial_{j}}^{1} \partial_{i}=C_{i j}^{k} \partial_{k} & \nabla_{\partial_{j}}^{3} \partial_{i}=\tilde{C}_{i j}^{k} \partial_{k} \\
S\left(\partial_{j}, \partial_{i}\right)=S_{i j}^{k} \partial_{k} & & T\left(\partial_{j}, \partial_{i}\right)=T_{i j}^{k} \partial_{k} .
\end{array}
$$

In the relation 3.6 let $X=\partial_{j}, Y=\partial_{i}$ and $Z=\partial_{l}$, then we can obtain the coefficients $C_{i j}^{k}$ and $\tilde{C}_{i j}^{k}$ as follows:

$$
\tilde{C}_{i j}^{k}=C_{i j}^{k}=\frac{1}{2} g^{l k}\left\{\partial_{j} g_{i l}+\partial_{i} g_{l j}-\partial_{l} g_{j i}+S_{j l}^{h} g_{i h}+S_{i j}^{h} g_{l h}-S_{l i}^{h} g_{j h}\right\}
$$

By attention to the relation 3.1, for computing the coefficients $F_{i j}^{k}$ and $\tilde{F}_{i j}^{k}$ we must consider four cases for any connection $\nabla^{a}$ as follows:

$$
\begin{aligned}
& F_{\alpha \beta}^{k}, F_{\alpha n+\beta}^{k}, F_{n+\alpha \beta}^{k}, F_{n+\alpha n+\beta}^{k} \\
& \tilde{F}_{\alpha \beta}^{k}, \tilde{F}_{\alpha n+\beta}^{k}, \tilde{F}_{n+\alpha \beta}^{k}, \tilde{F}_{n+\alpha n+\beta}^{k} .
\end{aligned}
$$


The Computation of $F_{\alpha \beta}^{k}$ and $\tilde{F}_{n+\alpha \beta}^{k}$.

In the equation 3.7 let $X=\delta_{\beta}, Y=\delta_{\alpha}$ and $Z=\delta_{\theta}$. Then we have,

$$
\begin{aligned}
2 F_{\alpha \beta}^{k} g_{k \theta}= & \delta_{\beta} g_{\alpha \theta}+\delta_{\alpha} g_{\theta \beta}-\delta_{\theta} g_{\beta \alpha}+T_{\beta \theta}^{h} g_{\alpha h}+T_{\alpha \beta}^{h} g_{\theta h}-T_{\theta \alpha}^{h} g_{\beta h} \\
2 \tilde{F}_{n+\alpha \beta}^{k} g_{k n+\theta}= & \delta_{\beta} g_{n+\alpha n+\theta}+\delta_{\alpha} g_{n+\theta}{ }_{n+\beta}-\delta_{\theta} g_{n+\beta}{ }_{n+\alpha} \\
& -T_{n+\beta n+\theta}^{h} g_{n+\alpha h}-T_{n+\alpha n+\beta}^{h} g_{n+\theta h}+T_{n+\theta n+\alpha}^{h} g_{n+\beta h} .
\end{aligned}
$$

Now for the same $X$ and $Y$ let $Z=\delta_{n+\theta}$, after some computations we have

$$
\begin{aligned}
2 F_{\alpha \beta}^{k} g_{k n+\theta}= & \delta_{\beta} g_{\alpha n+\theta}+\delta_{\alpha} g_{n+\theta \beta}+\delta_{n+\theta} g_{\beta \alpha} \\
& +T_{\beta n+\theta}^{h} g_{\alpha h}+T_{\alpha \beta}^{h} g_{n+\theta h}-T_{n+\theta \alpha}^{h} g_{\beta h} \\
2 \tilde{F}_{n+\alpha \beta}^{k} g_{k \theta}= & \delta_{\beta} g_{n+\alpha \theta}+\delta_{\alpha} g_{\theta}{ }_{n+\beta}-\delta_{n+\theta} g_{n+\beta} n+\alpha \\
& -T_{n+\beta}^{h} g_{n+\alpha}-T_{n+\alpha n+\beta}^{h} g_{\theta h}+T_{\theta n+\alpha}^{h} g_{n+\beta h} .
\end{aligned}
$$

Now the relations $(3.14,3.16)$ and the relations $(3.15,3.17)$ respectively show that

$$
\begin{aligned}
F_{\alpha \beta}^{k}= & \frac{1}{2} g^{k l}\left\{\delta_{\beta} g_{\alpha l}+\delta_{\alpha} g_{l \beta}+T_{\beta l}^{h} g_{\alpha h}+T_{\alpha \beta}^{h} g_{l h}-T_{l \alpha}^{h} g_{\beta h}\right\} \\
& -\frac{1}{2}\left(g^{k \gamma} \delta_{\gamma}-g^{k n+\gamma} \delta_{n+\gamma}\right) g_{\beta \alpha} \\
\tilde{F}_{n+\alpha \beta}^{k}= & \frac{1}{2} g^{k l}\left\{\delta_{\beta} g_{n+\alpha} l+\delta_{\alpha} g_{l n+\beta}-T_{n+\beta}^{h} g_{n+\alpha h}-T_{n+\alpha n+\beta}^{h} g_{l h}\right. \\
& \left.+T_{l n+\alpha}^{h} g_{n+\beta}\right\}-\frac{1}{2}\left(g^{k \gamma} \delta_{n+\gamma}+g^{k n+\gamma} \delta_{\gamma}\right) g_{n+\beta n+\alpha} .
\end{aligned}
$$

The Computation of $F_{\alpha n+\beta}^{k}$ and $\tilde{F}_{n+\alpha n+\beta}^{k}$.

Similar to the pervious case in the equation 3.7, let $X=\delta_{n+\beta}, Y=\delta_{\alpha}$ and $Z=\delta_{\theta}$. Then we have,

$$
\begin{aligned}
2 F_{\alpha n+\beta}^{k} g_{k \theta}= & \delta_{n+\beta} g_{\alpha \theta}-\delta_{\alpha} g_{\theta n+\beta}+\delta_{\theta} g_{n+\beta \alpha} \\
& -T_{n+\beta}^{h} g_{\alpha h}-T_{\alpha n+\beta}^{h} g_{\theta h}+T_{\theta \alpha}^{h} g_{n+\beta h} \\
2 \tilde{F}_{n+\alpha n+\beta}^{k} g_{k n+\theta}= & \delta_{n+\beta} g_{n+\alpha}{ }_{n+\theta}+\delta_{\alpha} g_{n+\theta \beta}-\delta_{\theta} g_{\beta n+\alpha} \\
& -T_{\beta n+\theta}^{h} g_{n+\alpha}-T_{n+\alpha \beta}^{h} g_{n+\theta h}+T_{n+\theta n+\alpha}^{h} g_{\beta h} .
\end{aligned}
$$

Now for $X=\delta_{n+\beta}, Y=\delta_{\alpha}$ let $Z=\delta_{n+\theta}$, then we have

$$
\begin{aligned}
2 F_{\alpha n+\beta}^{k} g_{k n+\theta}= & \delta_{n+\beta} g_{\alpha n+\theta}-\delta_{\alpha} g_{n+\theta n+\beta}-\delta_{n+\theta} g_{n+\beta \alpha} \\
& -T_{n+\beta n+\theta}^{h} g_{\alpha h}-T_{\alpha{ }_{n+\beta}}^{h} g_{n+\theta h}+T_{n+\theta \alpha}^{h} g_{n+\beta h} \\
2 \tilde{F}_{n+\alpha}^{k}{ }_{n+\beta} g_{k \theta}= & \delta_{n+\beta} g_{n+\alpha \theta}+\delta_{\alpha} g_{\theta \beta}-\delta_{n+\theta} g_{\beta n+\alpha} \\
& -T_{\beta \theta}^{h} g_{n+\alpha}-T_{n+\alpha \beta}^{h} g_{\theta h}+T_{\theta n+\alpha}^{h} g_{\beta h} .
\end{aligned}
$$

Now the relations $(3.20,3.22)$ and the relations $(3.21,3.23)$ respectively show that

$$
\begin{aligned}
F_{\alpha n+\beta}^{k}= & \frac{1}{2} g^{k l}\left\{\delta_{n+\beta} g_{\alpha l}-\delta_{\alpha} g_{l n+\beta}-T_{n+\beta}^{h} g_{\alpha h}-T_{\alpha n+\beta}^{h} g_{l h}+T_{l \alpha}^{h} g_{n+\beta h}\right\} \\
& +\frac{1}{2}\left(g^{k \gamma} \delta_{\gamma}-g^{k n+\gamma} \delta_{n+\gamma}\right) g_{n+\beta} \alpha \\
\tilde{F}_{n+\alpha n+\beta}^{k}= & \frac{1}{2} g^{k l}\left\{\delta_{n+\beta} g_{n+\alpha} l+\delta_{\alpha} g_{l \beta}-T_{\beta}^{h} g_{n+\alpha}-T_{n+\alpha \beta}^{h} g_{l h}\right. \\
& \left.+T_{l n+\alpha}^{h} g_{\beta}\right\}-\frac{1}{2}\left(g^{k \gamma} \delta_{n+\gamma}+g^{k n+\gamma} \delta_{\gamma}\right) g_{\beta n+\alpha} .
\end{aligned}
$$

The Computation of $F_{n+\alpha \beta}^{k}$ and $\tilde{F}_{\alpha \beta}^{k}$.

In 3.7 consider $X=\delta_{\beta}, Y=\delta_{n+\alpha}$ and $Z=\delta_{\theta}$. Then we have

$$
\begin{aligned}
2 F_{n+\alpha \beta}^{k} g_{k \theta}= & \delta_{\beta} g_{n+\alpha \theta}-\delta_{n+\alpha} g_{\theta \beta}-\delta_{\theta} g_{\beta}{ }_{n+\alpha} \\
& +T_{\beta \theta}^{h} g_{n+\alpha h}+T_{n+\alpha \beta}^{h} g_{\theta h}-T_{\theta n+\alpha}^{h} g_{\beta} \\
2 \tilde{F}_{\alpha \beta}^{k} g_{k n+\theta}= & \delta_{\beta} g_{\alpha n+\theta}+\delta_{n+\alpha} g_{n+\theta}{ }_{n+\beta}-\delta_{\theta} g_{n+\beta \alpha} \\
& -T_{n+\beta n+\theta}^{h} g_{\alpha h}-T_{\alpha n+\beta}^{h} g_{n+\theta h}+T_{n+\theta \alpha}^{h} g_{n+\beta h} .
\end{aligned}
$$


Now for the same $X$ and $Y$ let $Z=\delta_{n+\theta}$, then we have

$$
\begin{aligned}
2 F_{n+\alpha \beta}^{k} g_{k n+\theta}= & \delta_{\beta} g_{n+\alpha n+\theta}-\delta_{n+\alpha} g_{n+\theta \beta}+\delta_{n+\theta} g_{\beta n+\alpha} \\
& +T_{\beta{ }_{n+\theta}}^{h} g_{n+\alpha h}+T_{n+\alpha \beta}^{h} g_{n+\theta h}-T_{n+\theta n+\alpha}^{h} g_{\beta h} \\
2 \tilde{F}_{\alpha \beta}^{k} g_{k \theta}= & \delta_{\beta} g_{\alpha \theta}+\delta_{n+\alpha} g_{\theta}{ }_{n+\beta}-\delta_{n+\theta} g_{n+\beta \alpha} \\
& -T_{n+\beta}^{h} g_{\alpha h}-T_{\alpha n+\beta}^{h} g_{\theta h}+T_{\theta \alpha}^{h} g_{n+\beta} .
\end{aligned}
$$

Equations $(3.26,3.28)$ and equations $(3.27,3.29)$ respectively show that

$$
\begin{aligned}
F_{n+\alpha \beta}^{k}= & \frac{1}{2} g^{k l}\left\{\delta_{\beta} g_{n+\alpha l}-\delta_{n+\alpha} g_{l \beta}+T_{\beta l}^{h} g_{n+\alpha} h+T_{n+\alpha \beta}^{h} g_{l h}-T_{l n+\alpha}^{h} g_{\beta h}\right\} \\
& +\frac{1}{2}\left(g^{k n+\gamma} \delta_{n+\gamma}-g^{k \gamma} \delta_{\gamma}\right) g_{\beta}{ }_{n+\alpha} \\
\tilde{F}_{\alpha \beta}^{k}= & \frac{1}{2} g^{k l}\left\{\delta_{\beta} g_{\alpha l}+\delta_{n+\alpha} g_{l n+\beta}-T_{n+\beta}^{h} g_{\alpha h}-T_{\alpha n+\beta}^{h} g_{l h}\right. \\
& \left.+T_{l \alpha}^{h} g_{n+\beta h}\right\}-\frac{1}{2}\left(g^{k \gamma} \delta_{n+\gamma}+g^{k n+\gamma} \delta_{\gamma}\right) g_{n+\beta \alpha} .
\end{aligned}
$$

The Computation of $F_{n+\alpha n+\beta}^{k}$ and $\tilde{F}_{\alpha n+\beta}^{k}$.

Now for the last time in equation 3.7, let $X=\delta_{n+\beta}, Y=\delta_{n+\alpha}$ and $Z=\delta_{\theta}$. Then we have,

$$
\begin{aligned}
2 F_{n+\alpha n+\beta}^{k} g_{k \theta}= & \delta_{n+\beta} g_{n+\alpha \theta}+\delta_{n+\alpha} g_{\theta n+\beta}+\delta_{\theta} g_{n+\beta n+\alpha} \\
& -T_{n+\beta}^{h} g_{n+\alpha h}-T_{n+\alpha n+\beta}^{h} g_{\theta h}-T_{\theta n+\alpha}^{h} g_{n+\beta h} \\
2 \tilde{F}_{\alpha n+\beta}^{k} g_{k n+\theta}= & \delta_{n+\beta} g_{\alpha{ }_{n+\theta}}+\delta_{n+\alpha} g_{n+\theta \beta}-\delta_{\theta} g_{\beta \alpha} \\
& -T_{\beta n+\theta}^{h}{ }_{\alpha h}-T_{\alpha \beta}^{h} g_{n+\theta}{ }_{n}+T_{n+\theta \alpha}^{h} g_{\beta h} .
\end{aligned}
$$

Similar to the pervious cases for the same $X$ and $Y$, let $Z=\delta_{n+\theta}$, after some computations we have

$$
\begin{aligned}
2 F_{n+\alpha}^{k}{ }_{n+\beta} g_{k n+\theta}= & \delta_{n+\beta} g_{n+\alpha n+\theta}+\delta_{n+\alpha} g_{n+\theta} n+\beta \\
& -T_{n+\beta n+\theta}^{h} \delta_{n+\alpha h}-T_{n+\alpha n+\beta}^{h} g_{n+\beta} g_{n+\theta}+T_{n+\theta}^{h} \\
2 \tilde{F}_{\alpha n+\beta}^{k} g_{k \theta}= & \delta_{n+\beta} g_{\alpha \theta+\beta h}+\delta_{n+\alpha} g_{\theta \beta}-\delta_{n+\theta} g_{\beta \alpha}-T_{\beta \theta}^{h} g_{\alpha h} \\
& -T_{\alpha \beta}^{h} g_{\theta h}+T_{\theta \alpha}^{h} g_{\beta h} .
\end{aligned}
$$

Now by the equations $(3.32,3.34)$ and $(3.33,3.35)$ we respectively have

$$
\begin{aligned}
F_{n+\alpha n+\beta}^{k}= & \frac{1}{2} g^{k l}\left\{\delta_{n+\beta} g_{n+\alpha l}+\delta_{n+\alpha} g_{l n+\beta}-T_{n+\beta}^{h}{ }_{l} g_{n+\alpha} h\right. \\
& \left.-T_{n+\alpha n+\beta}^{h} g_{l h}+T_{l n+\alpha}^{h} g_{n+\beta}\right\} \\
& +\frac{1}{2}\left(g^{k \gamma} \delta_{\gamma}-g^{k n+\gamma} \delta_{n+\gamma}\right) g_{n+\beta} n+\alpha \\
\tilde{F}_{\alpha n+\beta}^{k}= & \frac{1}{2} g^{k l}\left\{\delta_{n+\beta} g_{\alpha l}+\delta_{n+\alpha} g_{l \beta}-T_{\beta l}^{h} g_{\alpha h}-T_{\alpha \beta}^{h} g_{l h}\right. \\
& \left.+T_{l \alpha}^{h} g_{\beta h}\right\}-\frac{1}{2}\left(g^{k \gamma} \delta_{n+\gamma}+g^{k n+\gamma} \delta_{\gamma}\right) g_{\beta \alpha} .
\end{aligned}
$$

Now by using the relations $J_{a} \circ v=h \circ J_{a}, v \circ J_{a}=J_{a} \circ h$ and 3.5 , we have

$$
\begin{aligned}
T^{D^{a}}(v X, v Y) & =\nabla_{v X} v Y-\nabla_{v Y} v X-[v X, v Y] \\
h T^{D^{a}}(h X, h Y) & =J_{a}\left(\nabla_{h Y} J_{a} h X-\nabla_{h X} J_{a} h Y+J_{a} h[h X, h Y]\right) .
\end{aligned}
$$

Suppose that $X, Y \in \Gamma\left(T \mathcal{U}^{\prime}\right)$ are two arbitrary vector fields on $\mathcal{U}^{\prime}$ which have the following representations in local coordinates:

$$
\begin{aligned}
& X=z^{\alpha} \delta_{\alpha}+z^{n+\alpha} \delta_{n+\alpha}+w^{\alpha} \partial_{\alpha}+w^{n+\alpha} \partial_{n+\alpha} \\
& Y=\tilde{z}^{\alpha} \delta_{\alpha}+\tilde{z}^{n+\alpha} \delta_{n+\alpha}+\tilde{w}^{\alpha} \partial_{\alpha}+\tilde{w}^{n+\alpha} \partial_{n+\alpha}
\end{aligned}
$$


After performing some computations we have:

$$
\begin{aligned}
T^{D^{a}}(v X, v Y) & =S(v X, v Y) \\
h T^{D^{a}}(h X, h Y) & =J_{a} T\left(J_{a} h Y, J_{a} h X\right) .
\end{aligned}
$$

The last equations show that $\nabla^{a}$ satisfies the second condition of the theorem.

In this step we show that $\nabla^{a}$ is unique. Let $\tilde{\nabla}^{a}$ be another linear connection on $V \mathcal{U}^{\prime}$ satisfying conditions (1) and (2).

By the equation $\left(\tilde{\nabla}_{v X}^{a} g\right)(v Y, v Z)=0$ we have

$$
v X(g(v Y, v Z))=g\left(\tilde{\nabla}_{v X}^{a} v Y, v Z\right)+g\left(v Y, \tilde{\nabla}_{v X}^{a} v Z\right),
$$

and so,

$$
\begin{aligned}
& v X(g(v Y, v Z))+v Y(g(v Z, v X))-v Z(g(v X, v Y))= \\
& \quad g\left(2 \tilde{\nabla}_{v Y}^{a} v X+T^{\tilde{D}^{a}}(v X, v Y)+[v X, v Y], v Z\right) \\
& \quad+g\left(T^{\tilde{D}^{a}}(v X, v Z)+[v X, v Z], v Y\right)+g\left(T^{\tilde{D}^{a}}(v Z, v Y)+[v Z, v Y], v X\right),
\end{aligned}
$$

on the other hand we have, $\left(\tilde{\nabla}_{h X}^{a}\right)\left(v J_{a} Y, v J_{a} Z\right)=0$, which shows that,

$$
\begin{aligned}
h X & \left(g\left(v J_{a} Y, v J_{a} Z\right)\right)+h Y\left(g\left(v J_{a} Z, v J_{a} X\right)\right)-h Z\left(g\left(v J_{a} X, v J_{a} Y\right)\right) \\
= & g\left(J_{a} h T^{\tilde{D}^{a}}(h X, h Y)+2 \tilde{\nabla}_{h Y}^{a} J_{a} h X+J_{a} h[h X, h Y], J_{a} h Z\right) \\
& +g\left(J_{a} h T^{\tilde{D}^{a}}(h X, h Z)+J_{a} h[h X, h Z], J_{a} h Y\right) \\
& +g\left(-J_{a} h T^{\tilde{D}^{a}}(h Z, h Y)-J_{a} h[h Z, h Y], J_{a} h X\right),
\end{aligned}
$$

where $\tilde{D}^{a}$ is the linear connection induced by $\tilde{\nabla}^{a}$ and theorem 3.1 .

The relations 3.45 and 3.46 show that $\tilde{\nabla}^{a}$ satisfies 3.6 and 3.7, respectively. Therefore $\nabla^{a}=\tilde{\nabla}^{a}$.

It is a natural question whether we can use $J_{2}$ to introduce a new connection similar to $J_{1}$ and $J_{3}$ ?

The answer is negative because $J_{2}$ dose not involve with the non-linear connection and we can not introduce a connection $D^{2}$ in a similar way to $D^{1}$ and $D^{3}$ but we can have the following theorem.

Theorem 3.3. Suppose that $\nabla$ is a Finsler connection on $\mathcal{U}^{\prime}$. Then the differential operator $D$ which is defined by

$$
D_{X} Y=\frac{1}{2}\left\{\nabla_{X} v Y-J_{1} \nabla_{X} J_{1} h Y-J_{2} \nabla_{X} J_{2} v Y-J_{3} \nabla_{X} J_{3} h Y\right\},
$$

where $X, Y \in \Gamma\left(T \mathcal{U}^{\prime}\right)$, is a linear connection on $\mathcal{U}^{\prime}$. Also $J_{1}, J_{2}$ and $J_{3}$ are parallel with respect to $D$.

Proof. For any $X, Y, Z \in \Gamma\left(T \mathcal{U}^{\prime}\right)$ and $f \in C^{\infty}\left(\mathcal{U}^{\prime}\right)$ we have

$$
\begin{aligned}
D_{f X+Y} Z= & \frac{1}{2}\left\{f \nabla_{X} v Z+\nabla_{Y} v Z-J_{1}\left(f \nabla_{X} J_{1} h Z+\nabla_{Y} J_{1} h Z\right)\right. \\
& \left.-J_{2}\left(f \nabla_{X} J_{2} v Z+\nabla_{Y} J_{2} v Z\right)-J_{3}\left(f \nabla_{X} J_{3} h Z+\nabla_{Y} J_{3} h Z\right)\right\} \\
= & f D_{X} Z+D_{Y} Z \\
D_{X}(f Y+Z)= & \frac{1}{2}\left\{X f(v Y)+f \nabla_{X} v Y+\nabla_{X} v Z-J_{1}\left(X f\left(J_{1} h Y\right)\right.\right. \\
& \left.+f \nabla_{X} J_{1} h Y+\nabla_{X} J_{1} h Z\right)-J_{2}\left(X f\left(J_{2} v Y\right)+f \nabla_{X} J_{2} v Y\right. \\
& \left.\left.+\nabla_{X} J_{2} v Z\right)-J_{3}\left(X f\left(J_{3} h Y\right)+f \nabla_{X} J_{3} h Y+\nabla_{X} J_{3} h Z\right)\right\} \\
= & \frac{1}{2}(2 X f(v Y+h Y))+f D_{X} Y+D_{X} Z \\
= & X f(Y)+f D_{X} Y+D_{X} Z .
\end{aligned}
$$

Therefore $D$ is a linear connection on $\mathcal{U}^{\prime}$.

Now we show that the almost hypercomplex structure is parallel with respect to $D$. 
Let $Y$ be a vector field on $\mathcal{U}^{\prime}$ which have the representation $Y=x^{\alpha} \delta_{\alpha}+y^{\alpha} \delta_{n+\alpha}+z^{\alpha} \partial_{\alpha}+w^{\alpha} \partial_{n+\alpha}$ in local coordinates. Then by a simple computation we have

$$
\begin{aligned}
\left(D_{X} J_{1}\right)(Y)= & D_{X}\left(J_{1}(Y)\right)-J_{1} D_{X} Y \\
= & \frac{1}{2}\left\{\nabla_{X} v J_{1} Y-J_{1} \nabla_{X} J_{1} h J_{1} Y-J_{2} \nabla_{X} J_{2} v J_{1} Y-J_{3} \nabla_{X} J_{3} h J_{1} Y\right. \\
& \left.-J_{1}\left\{\nabla_{X} v Y-J_{1} \nabla_{X} J_{1} h Y-J_{2} \nabla_{X} J_{2} v Y-J_{3} \nabla_{X} J_{3} h Y\right\}\right\} \\
= & 0
\end{aligned}
$$

In a similar way we can show $\left(D_{X} J_{2}\right)(Y)=\left(D_{X} J_{3}\right)(Y)=0$. Hence $J_{1}, J_{2}$ and $J_{3}$ are parallel with respect to $D$.

\section{References}

[1] Bejancu, A. and Farran, H. R. Geometry of Pseudo-Finsler Submanifolds, Kluwer Academic Publishers, 2000.

[2] Bejancu, A. and Farran, H. R. A comparison between the induced and the intrinsic Finsler connections on a Finsler submanifold, Algebras, Groups and Geometrie. 16(1999), no. 1, 11-23.

[3] Esrafilian, E. and Salimi Moghaddam, H. R. The Relation Between the Associate Almost Complex Structure to $H M^{\prime}$ and $\left(H M^{\prime}, S, T\right)$-Cartan Connections, SIGMA. 2(2006), 067, 7 pages.

[4] Gibbons, G. W. Papadopoulos, G. and Stelle, K. S. HKT and OKT geometries on soliton black hole moduli spaces, Nucl. Phys. B. 508(1997), 623-658.

[5] Poon, Y. S. Examples of Hyper-Kähler Connections with Torsion, Vienna, Preprint ESI, 770(1999), 1-7.

[6] Salimi Moghaddam, H. R. Randers Metrics of Berwald type on 4-dimensional hypercomplex Lie groups, J. Phys. A: Math. Theor. 42(2009), 095212(7pp).

[7] Salimi Moghaddam, H. R. On the Geometry of Some Para-Hypercomplex Lie Groups, Archivum Mathematicom BRNO. 45(2009), 159-170.

\section{Affiliations}

H. R. SALimi MOGHADDAM

ADDRESS: Department of Mathematics, Faculty of Sciences, University of Isfahan Isfahan-81746-73441- IRAN

E-MAIL: hr.salimi@sci.ui.ac.ir and salimi.moghaddam@gmail.com 